\title{
Successful Outcome of Post Liposuction Extensive Necrotizing Soft Tissue Infection of Abdomen and Concomitant Jejunal Perforation-A Case Report
}

\author{
Hardeep Singh ${ }^{1}$ Rakesh Kumar Khazanchi ${ }^{1}$ Sanjay Mahendru ${ }^{1}$ \\ ${ }^{1}$ Division of Plastic, Reconstructive and Aesthetic Surgery, \\ Medanta-The Medicity, Gurugram, India

\begin{abstract}
Address for correspondence Hardeep Singh, MCh, Department of Plastic, Reconstructive and Aesthetic Surgery, Medanta-The Medicity, Sector 38, Gurugram, Haryana 122001, India (e-mail: drhardeepaulakh@gmail.com).
\end{abstract}

Indian J Plast Surg 2019;52:238-241

\begin{abstract}
Keywords

- liposuction

Introduction Liposuction is one of the common cosmetic surgery procedures performed. Although rare, the complications associated with it are necrotizing soft tissue infection and bowel perforation. We would like to share our experience of such a complication that was managed successfully.

Case Report We were referred a 65-year-old male patient with signs and symptoms of intestinal obstruction who had undergone liposuction of abdomen 1 week before and now had discoloration of the abdominal skin. The discoloration was present from just below the costal margin and was extending up to bilateral inguinal regions. Laterally the discoloration extended up to the mid axillary line on both sides. Imaging studies showed dilated small bowel. During laparotomy, he underwent debridement of all discolored skin and repair of the single jejunal perforation. Postoperatively patient was first managed on intravenous fluids, nasogastric aspiration and total parenteral nutrition for 10 days. The wound was managed with silver dressings that led to healthy granulations. The patient was at high risk for anesthesia for skin grafting; hence, he was managed with allograft for 10 days. The patient then underwent skin grafting once he was fit. The graft took up well and he resumed regular activities.

- necrotising soft issue Conclusion This is unique as the patient had extensive necrotizing soft tissue infection of the abdominal skin after liposuction along with intestinal perforation and infection

- perforation obstruction that was managed successfully due to aggressive surgical intervention, allograft, and good supportive care both in the intensive care unit and in ward.
\end{abstract}

\section{Introduction}

Liposuction is the most commonly performed cosmetic surgery procedure worldwide. ${ }^{1}$ Although the results of liposuction are gratifying it is sometimes associated with complications even in experienced hands. Bowel perforation and resultant necrotizing soft tissue infection are two complications having a high morbidity and possible mortality. ${ }^{2}$ We present here a case report of post liposuction extensive necrotizing soft tissue infection of the abdominal skin due to bowel perforation with sepsis that was managed successfully.

\section{Case Report}

A 65-year-old male patient presented in the emergency department with history of abdominal pain, distension, obstipation, and discoloration of abdominal skin for 7 days. He had undergone liposuction of the abdomen 1 week back in another hospital. Patient also gave a past history of gastric bypass 7 years back for morbid obesity.

On clinical examination, the patient had tachycardia, tachypnoea, normal blood pressure, abdominal distension, and subcutaneous emphysema of abdominal skin. There was
DOI https://doi.org/

10.1055/s-0039-1696079

ISSN 0970-0358.
License terms

()(1) $\Theta \circledast$ 
blackish discoloration of the abdominal skin from just below the costal margin and extending up to bilateral inguinal region ( - Fig. $\mathbf{1}$ ). Laterally the discoloration extended up to the mid axillary line on both sides. The area of discoloration was surrounded by erythema all around. Diffuse tenderness was present all over the abdomen but there was no rebound tenderness. The contrast CT scan of the abdomen showed hold up of the contrast in the small intestine. The jejunum and the proximal ileal loops were dilated. There was no free fluid in the abdomen. There were specks of air in the abdomen.

The patient was taken for exploratory laparotomy. All the dead necrotic abdominal skin and the subcutaneous tissue were excised till the anterior rectus sheath that was found to be healthy. There was copious amount of seropurulent fluid in the perifascial planes. A mid ileal loop was kinked and was adhered to the duodenojejunal junction leading to obstruction. The obstruction was relieved. There was a single perforation in the mid jejunum that was repaired. After debridement the linea alba was closed with no. 1 loop nylon.

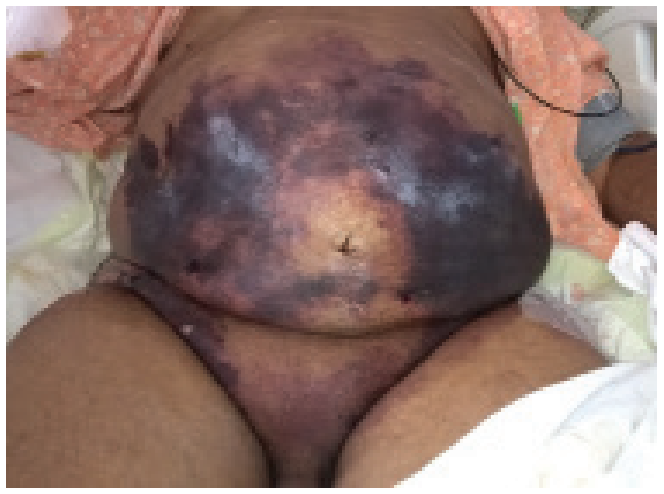

Fig. 1 Extensive discoloration of the abdominal skin.
Postoperatively patient was first managed on IV fluids, nasogastric aspiration, and total parenteral nutrition for 10 days. He developed altered sensorium, hyponatremia, and sepsis secondary to pneumonia which was managed with IV antibiotics according to the culture reports. The cultures showed growths of E. Coli and Enterococcus. The wound was managed with Acticoat (Smith \& Nephew, London, United Kingdom) dressings (-Fig. 2).

The condition of the wound improved in two weeks, granulations appeared, and the amount of soakage reduced (-Fig. 3). The patient was at high risk for skin grafting from anesthesia point of view, as he had intermittent fever and persistent tachycardia. Hence he was managed with an allograft for 10 days ( - Fig. 4). The application of the allograft reduced the exudate further and the dressing was changed once daily.

Four weeks after the initial debridement patient underwent autologous skin grafting. The graft took up well. He had uneventful recovery following skin grafting. The patient developed incisional hernia one year after skin grafting. He underwent incisional hernia repair at his hometown and he is back to regular activities, 2 years at follow-up ( - Fig. $\mathbf{5}$ ).

\section{Discussion}

The quest to reduce the excessive fat accumulated in the body has been there from nearly a century since 1921 when Charles Dujarier tried removing the excessive fat of the legs of a dancer. ${ }^{3}$ The complications also started right from the same case as the patient ended up having an amputation of the leg due to a femoral artery injury during the procedure. The German physician Schrudde started using a less invasive technique to remove fat in 1972 by using

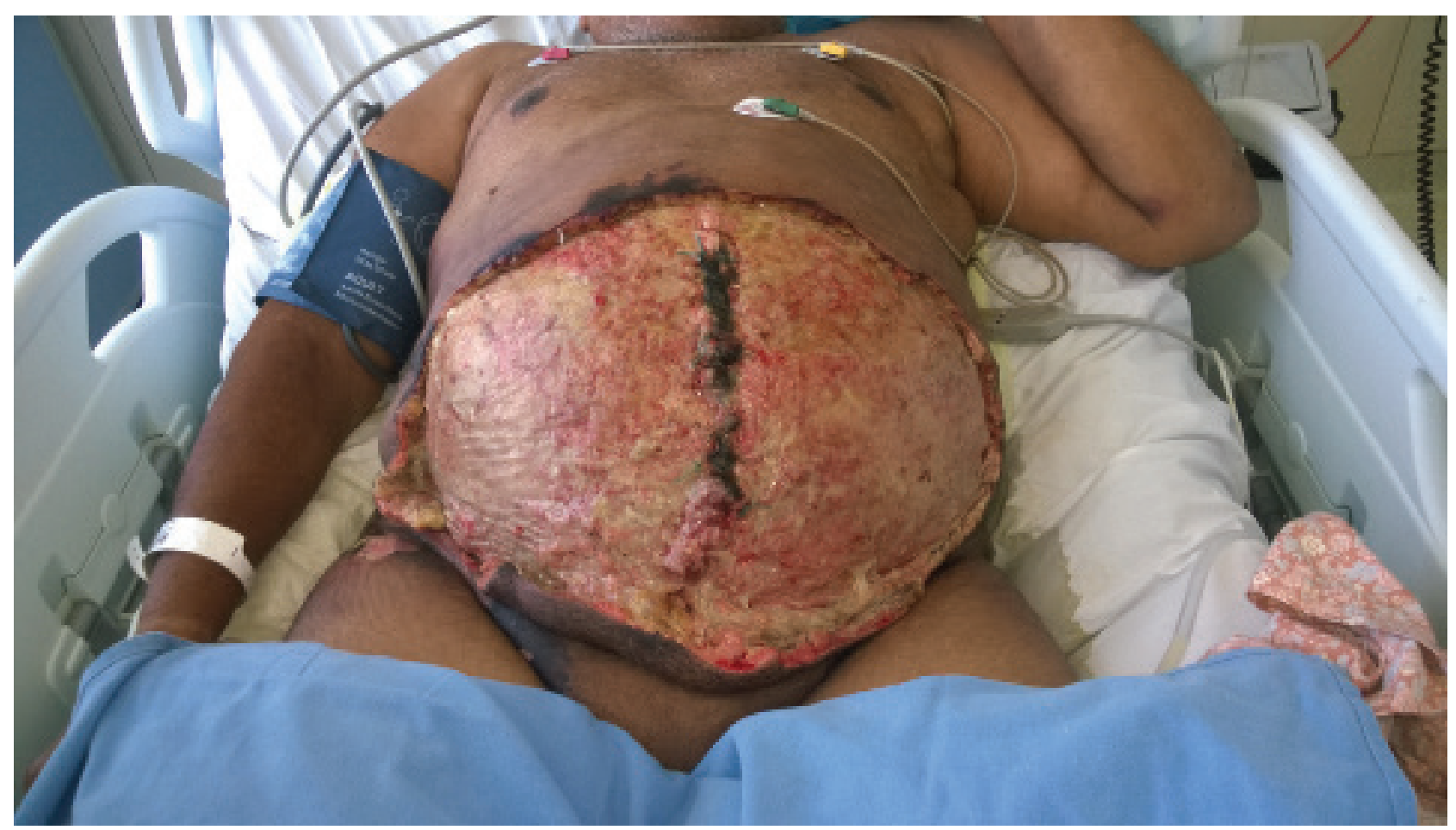

Fig. 2 Post debridement day 1. 


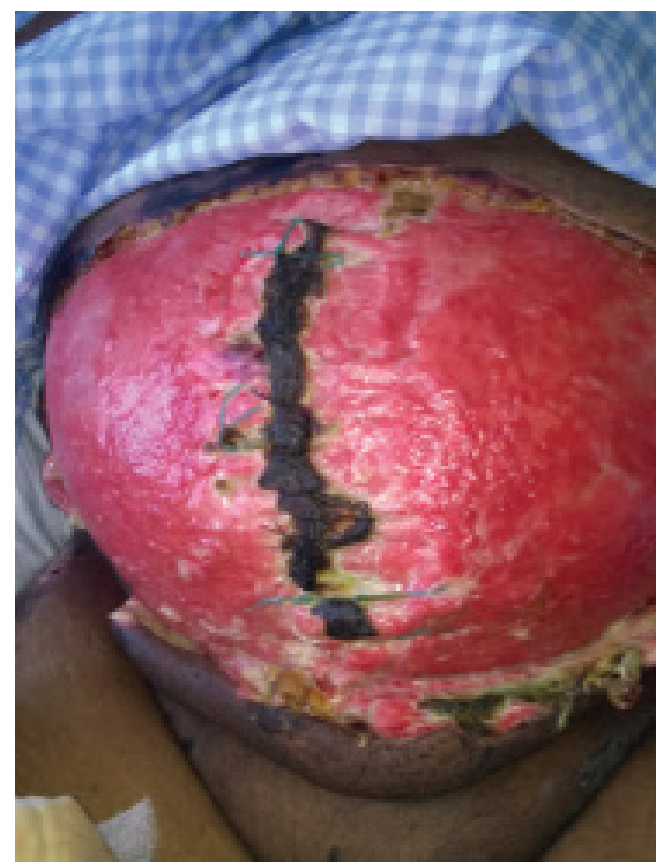

Fig. 3 Post debridement day 15 showing healthy granulation tissue.

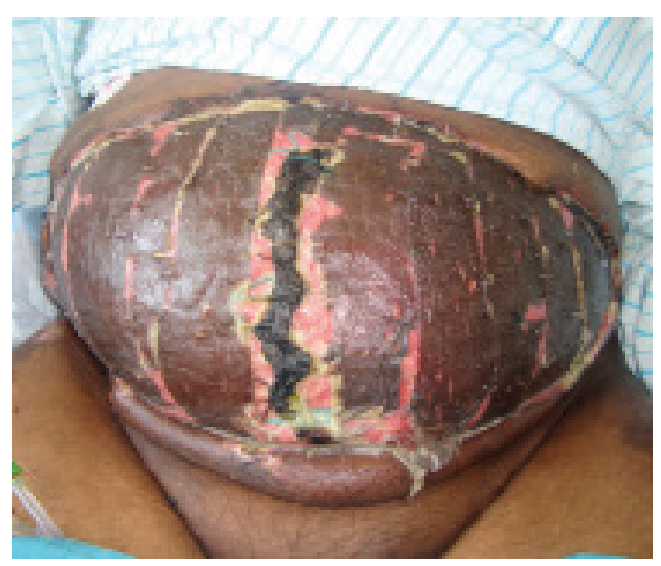

Fig. 4 Allograft application on raw area over abdomen.

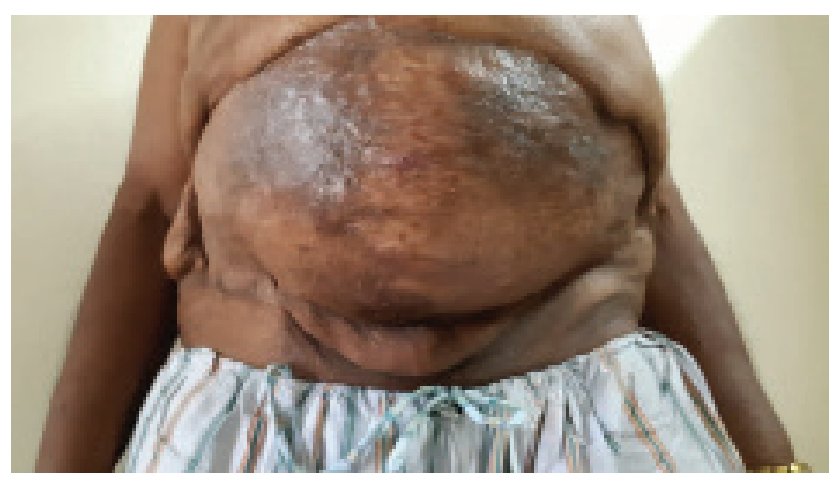

Fig. 5 Two years post skin grafting.

a uterine curette. Later on Illouz popularized liposuction (suction-assisted lipectomy) with his large series of cases. ${ }^{4}$

Complications associated with liposuction range from 8.6 to $20 \%$. These can be divided into local and systemic. The local complications include contour deformities, seroma, hematoma, hypertrophic scar, and asymmetry. The systemic complications are those having risk to life. The common systemic complications are bowel perforation, deep vein thrombosis, skin necrosis, sepsis, fat embolism, and hypothermia. ${ }^{5}$

Infection after liposuction is very rare with incidents in various author reports less than $1 \%{ }^{6}$ Not all infections post liposuction end up in necrotizing soft tissue infection but almost all are initially labeled as cellulitis.

The cardinal signs of necrotizing soft tissue infection are as follows:

1. Pain out of proportion to clinical signs.

2. Tender erythematous and swollen area beyond the zone of cellulitis.

3. Rapid change of skin color from red to purple with bullae formation.

The mortality rate in necrotizing fasciitis is very high ranging from 30 to $50 \%{ }^{3}$ Radical surgical debridement and empirical antibiotics is the first line of management in necrotizing soft tissue infection. The antibiotic needs to change according to the cultures. ${ }^{7}$

Perforation of an intraperitoneal organ though rare is also underestimated. ${ }^{1}$ The delay in diagnosis of this complication is the main cause of increased mortality associated with it. The perforation commonly occurs in patients with an abdominal wall weakness or diastases of rectus abdominis muscles, patients with umbilical hernia, and obese patients. ${ }^{8}$ Ileum is the most common site of perforation that classically presents with multiple perforations although single perforation is also not rare. ${ }^{1}$ The knowledge about the risk factors, early diagnostic imaging, and surgical intervention can save a life, which usually does not happen every time. Exploration of the wound and stoma are lifesaving many times. In our case, the patient had a single perforation in the jejunum, our general surgeons opted for closure of the perforation because the perforation was very proximal for any stoma, and there was no skin over the abdomen for the same. The contamination and spread of infection from this perforation in our case was contained probably because of previous bariatric surgery that had limited the peritonitis and systemic effects of it. However, the infection had spread to the parietes leading to necrotizing soft tissue infection of the abdominal wall.

Wound coverage may not be possible early in all the patients. Allograft can be temporarily used to tide over the crisis period and a definitive surgery can be planned in future when the patient's general condition settles down. The frequency of dressings also reduces, the overall effect on the patient's hyperdynamic state is beneficial. Allograft has been extensively used for raw areas secondary to burns, trauma, and Steven Johnson syndrome, but its use for covering the post liposuction extensive skin necrosis is not reported. Adherence of allograft to the raw area is an indirect indicator of healthy bed and we can predict good auto graft uptake. Negative pressure wound therapy (NPWT) was a valuable option that was considered before allograft application to tide over this period and keep the wound healthy. It was not used as the wound was extending in both inguinal regions; hence, getting a seal for negative suction was not possible. 
Simultaneous occurrence of these two complications can further worsen the situation. These systemic injuries can lead to multiple organ failure and is often fatal. This situation may need multiple sessions of debridement and laparotomy with the long intensive care unit (ICU) stay. The importance of the above mentioned procedure needs to be clearly conveyed to the relatives and honest disclosure to the patient as soon as the problem is detected is essential. A confident discussion on the measures to remedy those problems helps restore patient faith in their surgeon.

The unique features in our case are as follows:

1. Extensive raw area created as a result of this major complication of liposuction (from costal region to inguinal region) has been not reported in literature before.

2. Most of these extensive necrotizing fasciitis are fatal; we managed to resurface it successfully and save the patient.

3. It is the only case in literature where post liposuction raw area was temporarily managed with allograft till the condition of the patient was stable enough for auto grafting.

\section{Conclusion}

Liposuction complications although rare can happen. Thorough knowledge of high-risk patients, early of detection of complications, honest explanation to patient and relatives, prompt action, and good supportive care helps manage these complications successfully.

\section{Conflict of Interest}

None.

\section{References}

1 Zakine G, Baruch J, Dardour JC, Flageul G. Perforation of viscera, a dramatic complication of liposuction: a review of 19 cases evaluated by experts in France between 2000 and 2012. Plast Reconstr Surg 2015;135(3):743-750

2 Heitmann C, Czermak C, Germann G. Rapidly fatal necrotizing fasciitis after aesthetic liposuction. Aesthetic Plast Surg 2000;24(5):344-347

3 Grazer FM. Suction-assisted lipectomy, suction lipectomy, lipolysis, and lipexeresis. Plast Reconstr Surg 1983;72(5):620-623

4 Bellini E, Grieco MP, Raposio E. A journey through liposuction and liposculture: Review. Ann Med Surg (Lond) 2017;24:53-60

5 Dixit VV, Wagh MS. Unfavourable outcomes of liposuction and their management. Indian J Plast Surg 2013;46(2):377-392

6 Shiffman MA, Prevention and treatment of liposuction complications. In: Shiffman MA, Di Giuseppe A, eds. LiposuctionPrinciples and Practice. 1st ed. New York, NY: Springer; 2006;333-334

7 Sharma D, Dalencourt G, Bitterly T, Benotti PN. Small intestinal perforation and necrotizing fasciitis after abdominal liposuction. Aesthetic Plast Surg 2006;30(6):712-716

8 Igra H, Lanzer D, Avoiding complications. In: Hanke CW, Sattler G, eds. Liposuction. 1st ed. Philadelphia, PA: Saunders; 2005;131-134 